\title{
Hippocampal CA3 Region Predicts Memory Sequences: Accounting for the Phase Precession of Place Cells
}

\author{
Ole Jensen and John E. Lisman ${ }^{1}$ \\ Volen Center for Complex Systems \\ Brandeis University \\ Waltham, MA 02254
}

\begin{abstract}
Hippocampal recordings show that different place cells fire at different phases during the same theta oscillation, probably at the peak of different gamma cycles. As the rat moves through the place field of a given cell, the phase of firing during the theta cycle advances progressively. In this paper we have sought to determine whether a recently developed model of hippocampal and cortical memory function can explain this phase advance and other properties of place cells. According to this physiologically based model, the CA3 network stores information about the sequence of places traversed during learning. Here we show that the phase advance can be understood if it is assumed that the hippocampus is in a recall mode that operates when the animal is already familiar with a path. In this mode, sensory information about the current position triggers recall of the upcoming 5-6 places (memories) in the path at a rate of one memory per gamma cycle. The model predicts that the average phase advance will be one gamma cycle per theta cycle, a value in reasonable agreement with the data. The model also correctly accounts for $(1)$ the fact that the firing of a place cell occurs during $\sim 7$ theta cycles (on average) as the animal crosses the place field; (2) the observation that the phase of place cell firing depends more systematically on position than on time; and (3) the fact that traversal of an already familiar path produces further modifications (shifts the firing of a cell to an earlier position in the path). This later finding suggests that recall
\end{abstract}

${ }^{1}$ Corresponding author. of previously stored information strengthens the memory of that information. In the model, this occurs because of a novel role of $N$-methyl-D-aspartate channels in recall. The general success of the model provides support for the idea that the hippocampus stores sequence information and makes predictions of expected positions during gamma-frequency recall.

\section{Introduction}

In previous papers in this series, we have examined how long-term memory can form in networks where memories are multiplexed by theta/ gamma oscillations (Jensen and Lisman 1996a,b, this issue; Jensen et al., this issue). A key conclusion is that the time constant of deactivation of the $N$-methyl-D-aspartate (NMDA) channels that govern synaptic modification in recurrent collaterals is an important determinant of the function of the network. If the time constant is shorter than the period of a gamma cycle, the network will perform an autoassociative function, linking different elements of the same memory. If the time constant is longer than the period of a gamma cycle, the network will perform a heteroassociative function, linking cells that represent different, temporally separated memories. We showed that these heteroassociative linkages are well suited for the storage and recall of memory sequences (Jensen and Lisman 1996b, this issue). The NMDA channels in the recurrent collaterals of the hippocampal CA3 region have a deactivation time constant of 100150 msec (Debanne et al. 1995), a time considerably longer than that of a gamma cycle. The implication is that the CA3 network is not an autoassociative network, as previously thought, but rather a heteroassociative network. This is an idea that others have recently proposed (Buzsáki 1989;

LEARNING \& MEMORY 3:279-287 (C) 1996 by Cold Spring Harbor Laboratory Press ISSN1072-0502/96 \$5.00

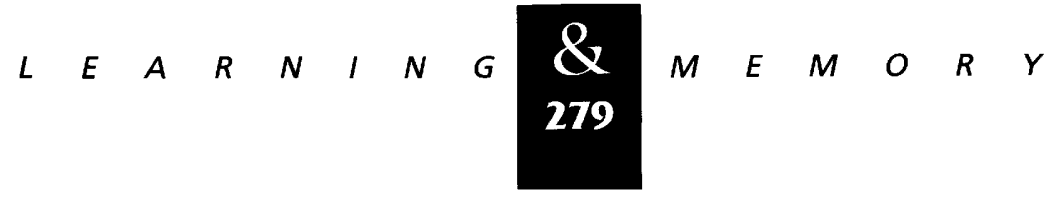


Manai and Levy 1993; Granger et al. 1994; Prepscius and Levy 1994; Blum and Abbott 1996) and there is some direct experimental support (Skaggs and McNaughton 1996).

In this paper we have sought to determine whether the specific model for storage and recall of sequence information developed in the previous papers can account for some of the key results regarding hippocampal place cells. These cells fire when the animal enters a subregion of an environment (O'Keefe and Dostrovsky 1971; Olton et al. 1978; McNaughton et al. 1983; Muller et al. 1987). A particularly dramatic finding is the importance of the phase of cell firing during the theta cycle: The phase systematically advances as the animal moves through its place field (O'Keefe and Recce 1993). A second result relates to the fact that the animal may not be moving at constant velocity. The results show that when velocity is not constant, position is a better predictor of phase than time (O'Keefe and Recce 1993). A third result is that the firing of place cells has been found to predict future locations (Muller and Kubie 1989). Finally, M.R. Metha, B.L. McNaughton, C.A. Barnes, N.S. Suster, K.L. Weaver, and J.L. Gerrard (pers. comm) have found that the traversal of a welllearned path leads to a progressive increase in the size of place fields and an offset in their position.

\section{Results and Discussion}

The model we have used to explain place cell data is described in the previous paper (Jensen and Lisman 1996b, this issue). In the current context we assume that the different memories being processed by the rat are the memories of specific places on the linear track. We assume that the system has both learning and recall modes. In learning mode, a cortical network acts as a shortterm buffer. It absorbs incoming information in real time, stores sequential memories (places) in adjacent gamma cycles, and produces repetition of the memories once every theta cycle in a timecompressed way. Signals from his "sample-andhold" buffer provide input into the hippocampus and drive synaptic modification in the recurrent collaterals of CA3. The synaptic modification in CA3 is governed by slow NMDA channels (deactivation time constant $\sim 150 \mathrm{msec}$ ) and the information encoded is the sequence of places along the track. In recall mode, the sensory information about the current place provides input to CA3 cells at the beginning of a theta cycle. This triggers CA3 through collateral connections to produce readout of the subsequent parts of the stored sequence at a rate of one memory (place) per gamma cycle. A novel aspect of the recall mechanisms we have proposed is that the CA3 cells encoding one memory excite the cells encoding the next memory by NMDA-mediated excitatory postsynaptic potentials (EPSPs). We found that this mechanism provided the delay needed for the sequence to be recalled at one memory per gamma cycle. In recall mode, the hippocampus serves as a predictor of the path to come.

The hippocampal recordings of O'Keefe and Recce (1993) and of Skaggs et al. (1996) were obtained as the rat moved along a one-dimensional track. Traversal of this track was repeated many times in the process of learning and data collection. Because the rat was familiar with the track, we assume that the rat is in recall mode. The phase advance of a place cell can be accounted for in terms of the diagram in Figure 1. Consider the path along the linear track to be a sequence of discrete

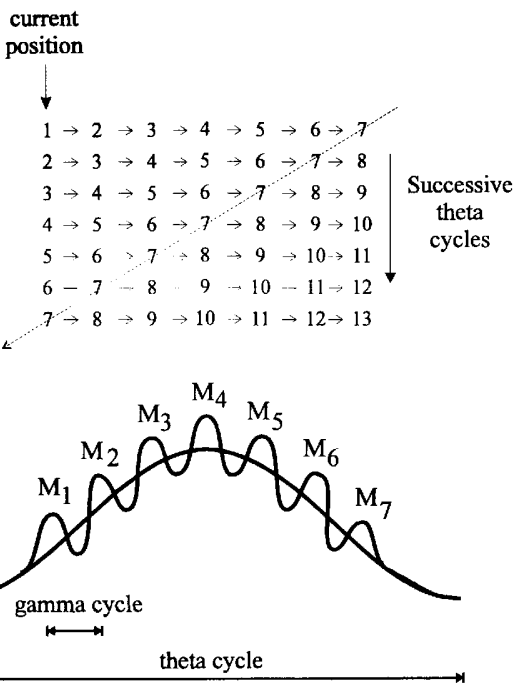

Figure 1: Diagrammatic illustration of how phase advance occurs as an animal moves through the place field on a well-known path. We assume that the animal has already learned the sequence of places on the path designated by the numerals $1-13$. As the animal moves through the place field, the sensory inputs regarding the current position are input to the CA3 region of the hippocampus at the beginning of each theta cycle. This input stimulates recurrent collaterals to produce firing of cells that encode subsequent positions in this sequence (path). It can be seen that the cell encoding position 7 fires earlier on each successive theta cycle.

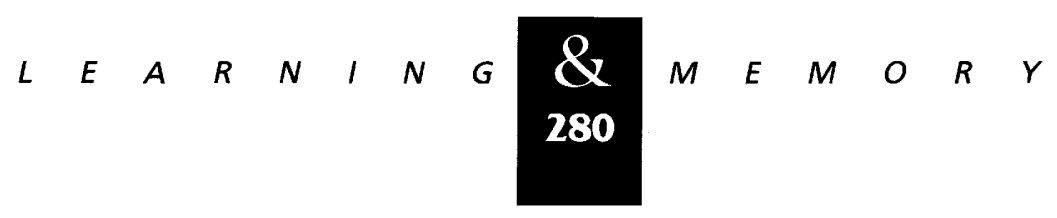


memories corresponding to positions 1-7 learned during the many previous traversals of the track. We assume that when the rat is running at its typical velocity during learning, it stores information about its current position in each theta cycle. During recall, when the animal enters position 1 , the sensory information about current position enters CA3 early during a theta cycle. This information serves as a cue to trigger the recall of the subsequent 6 memories (positions) in the stored sequence (positions 2-7), each in a different gamma subcycle of a theta period. As the animal moves along the linear track, the current position changes. Thus when the next theta cycle occurs, the sensory input to the hippocampus will be position 2 in the sequence. This input then stimulates recall of positions 3-8 in subsequent gamma cycles. This process continues during subsequent theta cycles. As can be seen in the example in Figure 1, the cell representing position 7 starts firing late in the theta cycle and systematically advances in phase as the animal moves through its place field. This provides a simple explanation of the phase advance observed in hippocampal recordings.

Figure 2 shows how the phase advance can occur in simulations of the CA3 network. The details of the simulation are given in the Methods section of the first paper in this series (Jensen et al., this issue). In these simulations we use a compound cue of two positions to represent the current position of the rat for reasons that will be discussed later ("Timing of Inputs to CA3"). Prior to the simulation a sequence of positions has been stored according to the method described in Jensen and Liman (1996b, this issue; Fig. 2). This is illustrated by the asymmetric synaptic matrix in Figure 2A. The simulations show the phase advance of place cell 7 (Fig. 2B) with respect to location. The plot of phase as a function of time is shown in Figure 2C.

The model predicts a phase advance of one gamma cycle per theta cycle and it is of interest to compare this with the observed data. This prediction holds only if the rat is moving as fast during recall as it did during learning, an assumption that may not be true on individual trials, but is probably true on average. From the data of O'Keefe and Recce (1993) we measured phase advances of $\sim 20^{\circ} / \mathrm{cm}$ of movement. The phase advance of one gamma cycle per theta cycle can be compared with this measured value as follows: The duration of one gamma cycle corresponding to $50^{\circ}$. The
A

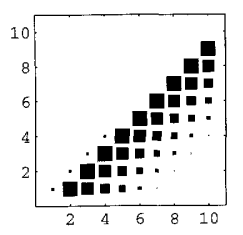

B
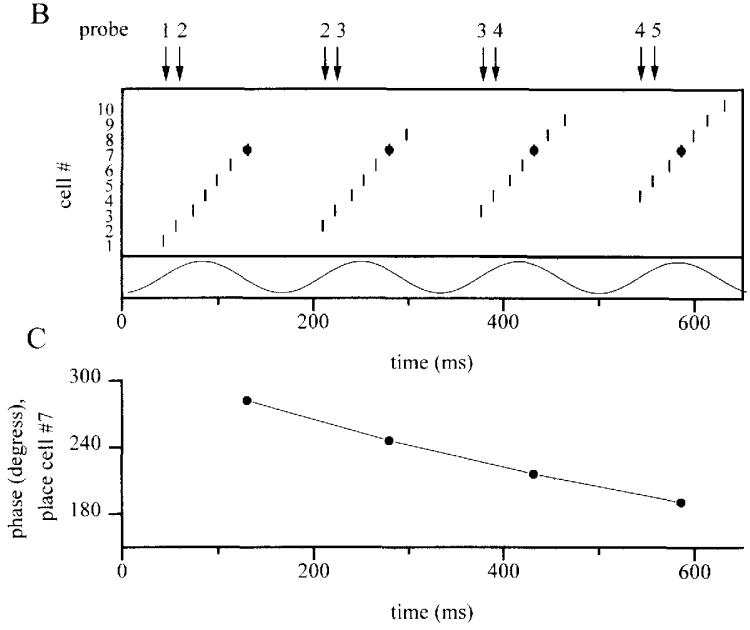

Figure 2: Simulation of place cell firing as the animal moves through its place field. Two positions near the current position of the rat are used to cue recall. $(A)$ Synaptic matrix of stored path; $(B)$ firing in seven representative cells is shown. Note that the firing in cell 7 (black dot) starts with late phase during the theta oscillation (shown at bottom) and moves to earlier phase as the animal moves forward in its place field. (C) Plot of phase of firing of cell 7 as a function of time. The rat is assumed to be moving at constant velocity. See Jensen and Lisman (1996b, this issue) and Jensen et al. (this issue) for a detailed description of the network. The parameters are the following: $V_{\text {rest }}=-60 \mathrm{mV}, V_{\text {thres }}=$ $-50 \mathrm{mV}, \mathrm{A}_{\mathrm{A}}=-150 \mathrm{pA}, \tau_{\mathrm{A}}=50 \mathrm{msec}, \mathrm{A}_{\mathrm{GABA}}=-150$ $\mathrm{pA}, \tau_{\mathrm{GABA}}=4.0 \mathrm{msec}, \mathrm{A}_{\mathrm{NMDA}}=784 \mathrm{pA}, \tau_{\mathrm{NMDA}, \mathrm{r}}=5.0$ $\mathrm{msec}, \tau_{\mathrm{NMDA}, \mathrm{f}}=50.0 \mathrm{msec}, \tau_{\text {post }}=2.0 \mathrm{msec}, \tau_{\text {delay }}=$ $0.5 \mathrm{msec}, \tau_{n p p}=1800 \mathrm{msec}, \tau_{\mathrm{pnp}}=36000 \mathrm{msec}, \tau_{\mathrm{pp}}=$ $900 \mathrm{msec}, \mathrm{f}_{\text {theta }}=6.0 \mathrm{~Hz}$, and $B_{\text {theta }}=150.0 \mathrm{pA}$. Note that we allow only synaptic modification in the simulations during recall only in Fig. 5. The synaptic values (A) ranged from 0 to 0.25 .

distance moved in one theta cycle is $\sim 4 \mathrm{~cm}$, as judged from the rat velocity plots of O'Keefe and Recce (1993) and their measurements of theta frequency of $\sim 10 \mathrm{~Hz}$. Thus, one gamma cycle per theta cycle translates roughly into a phase advance of $50 \% / 4 \mathrm{~cm} \sim 12 \% \mathrm{~cm}$. This is in reasonable agreement with the measured value of $20 \% \mathrm{~cm}$.

A closely related comparison of theory and model can be made. The model predicts that on average a place cell will fire for seven theta cycles 
as the animal moves through the place field. Both the records of O'Keefe and Recce (1993, Figs. 2,5, and 6) and Skaggs et al. (1996, Fig. 6A-D) show that this number is approximately correct. In the paper of Skaggs et al. (1996), Figure 6A-D illustrates the spike histogram of a place cell as a function of theta cycles binned over multiple runs. If we count only bins that have activity $>10 \%$ of the maximum activity, we find that a place cell is active on $10.3 \pm 1.3$ theta cycles $(N=4)$. If we count only bins having $>20 \%$ of the maximum activity, the value is $8.8 \pm 1.3(N=4)$. If only bursts rather than spikes are taken into account these numbers would probably be smaller (see Discussion).

The model makes the further prediction that the number of cycles during which firing occurs should be inversely related to the velocity of the rat during recall. If the velocity of the animal is the same during recall as during learning firing should occur on seven theta cycles. Furthermore, the size of the place field should be the velocity of the rat times the duration of seven theta cycles. For a velocity of $0.4 \mathrm{~m} / \mathrm{s}$ and a theta frequency of $10 \mathrm{~Hz}$, the predicted place cell size will be $\sim 30 \mathrm{~cm}$. This is in the right range. The model makes the further prediction that the size of the place fields should be related to the average velocity of the rat during learning. These predictions remain to be tested.

O'Keefe and Recce (1993) were interested in whether the phase of firing was more linear if they plotted phase as a function of time or position. Time and position are not proportional because the rat does not move at constant velocity. They found that the phase advanced more linearly with position than with time. In Figure 3, we show the phase as a function of time (Fig. 3A) and position (Fig. 3B) for three different velocities. The slope of the phase advance is independent of velocity when plotted as a function of position, but not when plotted as a function of time. The model thus correctly predicts that for a rat moving at varying velocity, the data points in a phase versus time plot will be much more scattered than in a phase versus position plot.

When the animal goes through a familiar environment, a CA3 cell will first fire with late phase because it represents a prediction of an expected position. Under these conditions it is being excited by other CA3 cells. As time passes and the animal moves forward, it will reach a place that corresponds to its actual position. Under these conditions it is being excited by direct sensory input. Given that the expectation of a future posi-
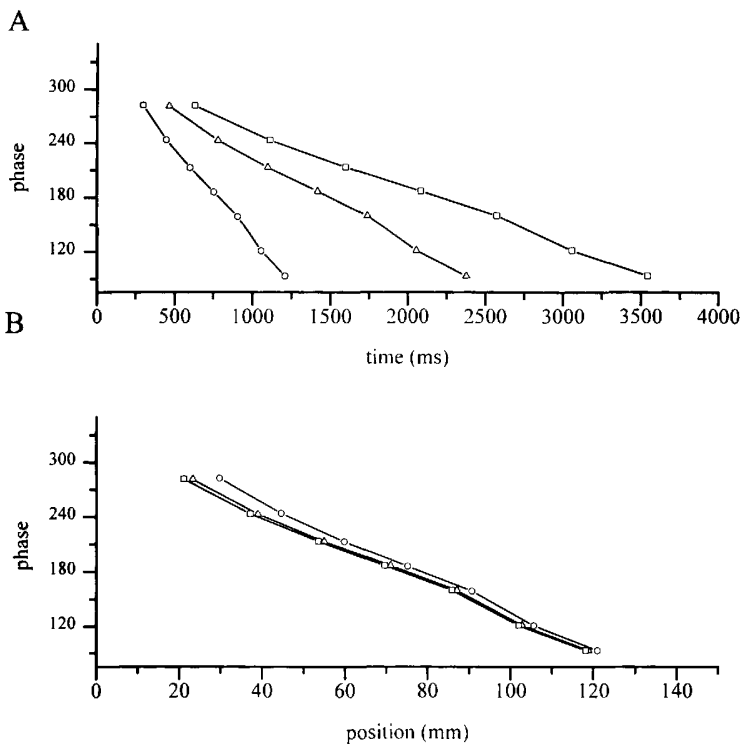

Figure 3: Effect of velocity on the change in phase as the animal moves through its place field. (A) When phase is plotted vs. time, the slope of the curve is affected by velocity $(O) v=100 \mathrm{~cm} / \mathrm{sec},(\triangle) v=50 \mathrm{~cm} /$ sec, $(\square) v=33 \mathrm{~cm} / \mathrm{sec}$; $(B)$ when plotted vs. position, the slope of the curve is unaffected by velocity $(O)$ $v=100 \mathrm{~cm} / \mathrm{sec},(\triangle) v=50 \mathrm{~cm} / \mathrm{sec},(\square) v=33 \mathrm{~cm} / \mathrm{sec}$.

tion is inherently more unreliable than a direct response to sensory cues about current position, cells that fire late in the theta cycle should be poorer predictors of position than cells that fire early in the theta cycle. Precisely this kind of relationship has been reported by Skaggs et al. (1996).

\section{LEARNING AND RECALL MODE}

We can give only a crude account of what determines whether the animal will be in learning or recall mode. It seems likely that this would depend strongly on the familiarity of the sensory input (see also Hasselmo and Bower 1993). In an unfamiliar environment, the animal would go into learning mode. In a familiar environment, the animal would be in recall mode and make predictions about future positions.

The model predicts that the position of a place field should be strongly affected by which mode the animal is in. In learning mode (Jensen and Lisman 1996a,b, this issue; Jensen et al., this issue), the hippocampus together with the cortical areas are operating in a sample-and-hold, short-term memory mode. Multiple sequential aspects of the sensory world are captured by a cortical network 
in real time; each memory is stored in an appropriate gamma slot, and repeated in compressed time at gamma frequency, once every theta cycle. This repetition occurs after the sensory input and provides the repetition necessary to drive synaptic modification in the recurrent synapses. This learning mode of operation is illustrated in Figure 4 (bottom; note that only four of seven gamma cycles are shown). In recall mode (Fig. 4, top), the hippocampus is not doing sample-and-hold, but rather is using current information to predict the future based on previously stored information. Figure 4 emphasizes the enormous difference in position of the place field, depending on what mode the animal is in. This shift should be equal to the size of a place field, that is, $\sim 20-30 \mathrm{~cm}$. Whether such large shifts occur remains to be determined. One way of detecting such shifts might be by placing a novel item on a well-known track. If the animal shifted to learning mode, there should be a large shift in the place field. A second approach would be to compare the position of a place field on a novel track with the position after the same track had become highly familiar.

Our ideas about how learning mode works are less well defined than for recall mode. The network might work in a continuous way in sampling the environment. In computer terms, the buffer could work as a top-down stack, with each new memory forcing out the oldest. In our investigations, we have not succeeded in finding a mechanistically plausible way of implementing such a stack. Alternatively, learning mode might involve a discontinuous process in which the sample-and- hold buffer operated until it became filled with seven memories. Input of new information might then cease until the system is cleared and reset. If the buffer works in this way no phase advance is expected during learning mode (Fig. 4).

Observations about rats as they learn about a novel environment suggest that the learning process is discontinuous. Eilam and Golani (1989) have found that when a rat is exploring a novel environment it will establish one or two home bases to which it always returns after an explorative excursion. Furthermore, when the rat is exploring, it alternates between progression and stopping (Golani et al. 1993; Tchernichovski and Golani 1995). The number of stops made within one excursion are uniformly distributed, with an upper bound of $10 \pm 2$. We hypothesize that each time a rat makes a stop within an excursion, a representation of that place is loaded into the sample-and-hold buffer. Perhaps this upper bound of the number of stops reflects the small number of memories that can be held in the sample-and-hold buffer (Lisman and Idiart 1995; Jensen and Lisman 1996a,b, this issue; Jensen et al., this issue).

\section{ROLE OF NMDA CHANNELS IN RECALL: RECALL PRODUCES LEARNING}

A dramatic prediction of the postulated role of NMDA channels in recall is that the act of recalling memories should strengthen them. This strengthening will be selective for the synapses that were originally strengthened in learning mode. Our sim-
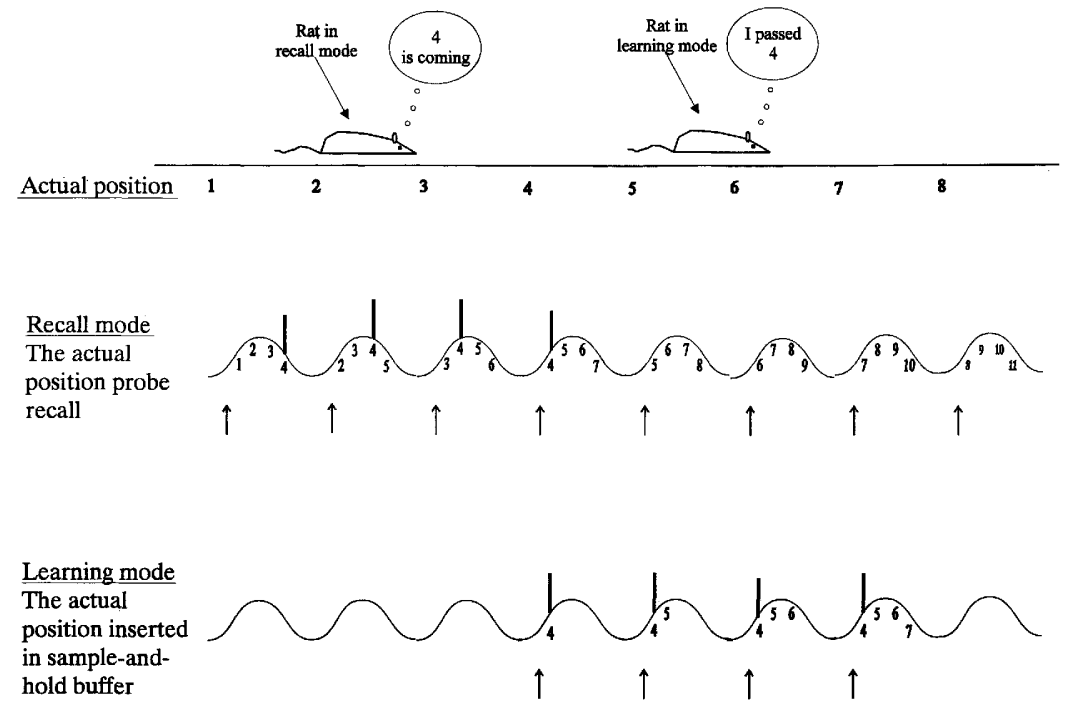

Figure 4: Diagrammatic illustration of the spatial dependence of place cell firing in learning mode and recall mode. Note that only four of the seven gamma slots are shown. The arrows mark the cue that provides information about current position to CA3. The numbers underneath the theta oscillation indicate the time of firing (in some cell) of the memory of the position designated by the numeral. The spikes indicate when spiking occurs in a particular cell. In learning mode we assume the buffer to be empty before information is introduced. The firing of the place cell occurs before reaching position 4 when the rat is in recall mode. The firing of the same cell occurs after position 4 when the rat is in learning mode.

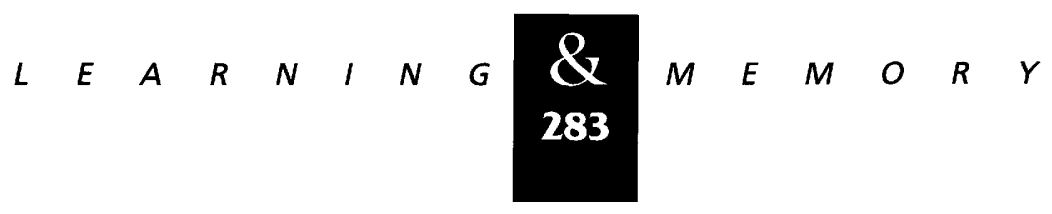


ulations show that this should lead to some surprising consequences. Because NMDA-mediated synaptic transmission is enhanced as the animal reexperiences a known path, EPSPs will become larger. Because it is these EPSPs that make cells fire, the inhibitory postsynaptic potentials (IPSPs) that separate gamma cycles will be more easily overcome by the EPSP; specifically, less of the IPSP will have to decay for the cell to reach threshold. As a result, cells will fire earlier in the gamma cycle. Because inhibition in the model is stimulated by the firing of pyramidal cells, negative feedback inhibition will arrive earlier. The overall consequence will be to increase the frequency of the gamma oscillations and thus to increase the number of gamma cycles that can fit into a theta cycle. This is illustrated in Figure 5. It can be seen that in lap 1 there are seven gamma cycles in a theta cy. cle. But as the animal does increasing laps in this known environment, continued use of the NMDA channels increases synaptic strengths (see the weight matrix) and eventually results in a situation

where gamma is so fast that as many as nine gamma cycles can occur in a theta cycle. This means that prediction occurs further ahead, that is, that the position where firing first occurs shifts in the direction opposite the movement (Fig. 5B). Furthermore, the overall size of the place field also gets larger (Fig. 5C). Because the period of gamma oscillations decreases when the rat reexperiences the known path, the phase advance per theta cycle (e.g., the slope of phase vs. position) will decrease.

Recent experiments by M.R. Mehta, B.L. McNaughton, C.A. Barnes, M.S. Suster, K.L. Weaver, and J.L. Gerrard (pers. comm.) appear to have been done under conditions appropriate for comparison with the predictions of Figure 5. Place cells were monitored as the rat ran through what was already a highly familiar track (the animal had run the same track on many previous days). It was observed that, as the animal did repeated laps, there were small progressive changes in the properties of place fields of the same order as those in Figure 5. It should be emphasized that these shifts

A
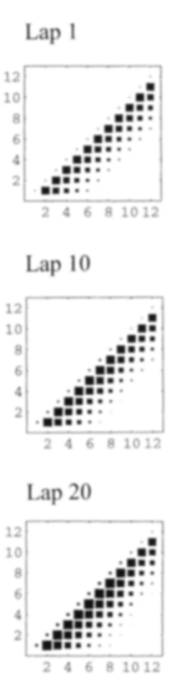
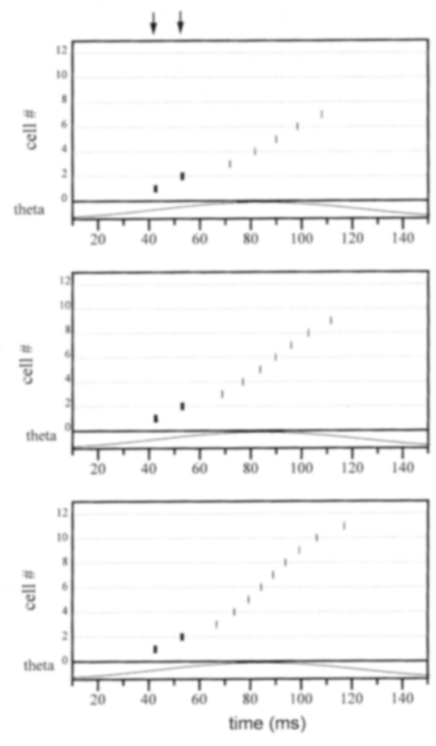

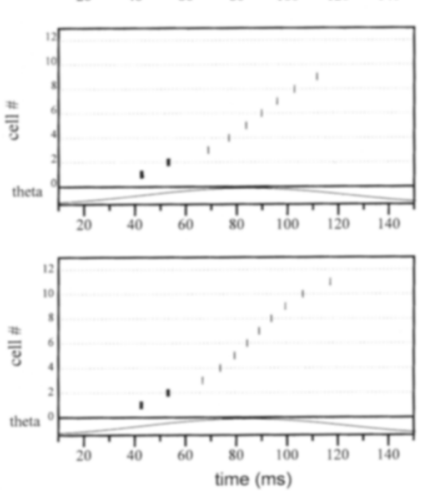

B

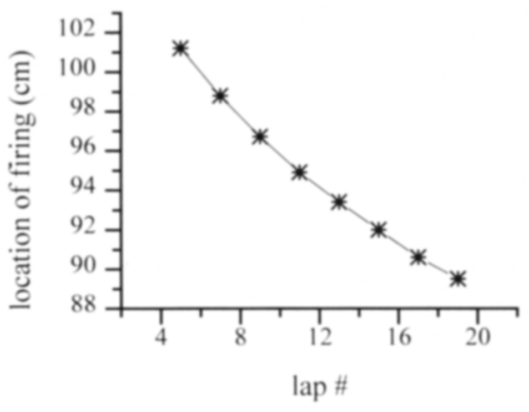

$$
\text { हิ }
$$
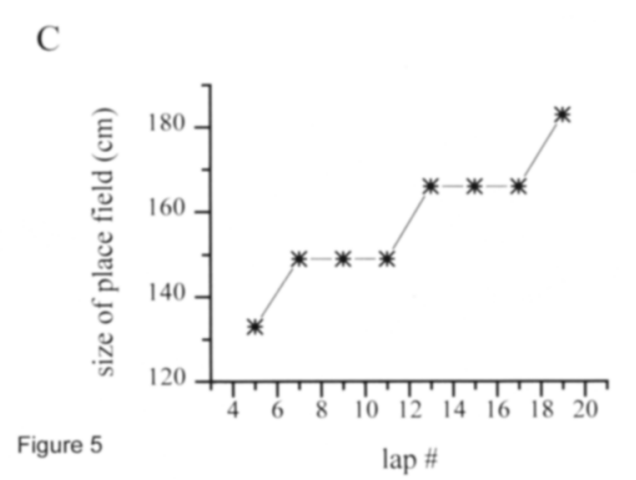

Figure 5: Simulations demonstrating that using the NMDA channel during recall mode produces further learning $(A)$ Plot of cell firing during traversal of a well-known path with animal in recall mode. Plots are shown after 1, 10, and 20 laps. Note the increase in number of gamma cycles. Synaptic matrices show that the synapses have become stronger. With increasing lap number, $(B)$ the time of occurrence of the firing of cell \#7 gets earlier on the track and $(C)$ there is an increase in the size of the place field. Velocity of rat: $V=100.0 \mathrm{~cm} / \mathrm{sec}$.

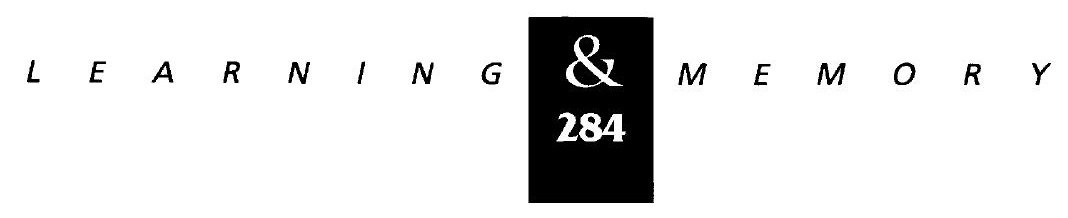


that occur in recall mode are much smaller than those we would predict by comparing learning mode with recall mode (see above and Fig. 4).

\section{THE POSTULATED ROLE OF NMDA CHANNEIS IN RECALL CAN BE DIRECTLY TESTED}

Predictions that could be tested are as follows:

1. NMDA channel blockers should block the phase advance seen as the rat moves through its place field.

2. NMDA channel blockers should block progressive changes in the size and position of the place field as the animal does laps on a wellknown track.

3. In a suitable behavioral test of sequence learning (perhaps path learning), NMDA channel blockers should reversibly block expression of previously learned knowledge about the sequence.

One complication of these experiments should be mentioned: Because an NMDA channel mediates excitation to interneurons, NMDA antagonists may produce disruption of hippocampal processing through action on interneurons rather than recurrent synapses.

WHY IS PHASE ADVANCE DATA SO NOISY COMPARED WITH THE PREDICTIONS OF THE MODEL?

The plots of phase versus position (O'Keefe and Recce 1993) based on experimental data are much noisier than our simulations (Fig. 2). This raises the question of whether brain networks work with the kind of precision envisioned in our model. This question cannot yet be answered, but we suggest two relevant considerations. First, the data plots are necessarily made by averaging together many trials. However, as now demonstrated directly (M.R. Mehta, B.L. McNaughton, C.A. Barnes, M.S. Suster, K.L. Weaver, and J.L. Gerrad, pers. comm.), the data is not stationary over such trials. This nonstationarity may obscure the regularity of underlying mechanisms. Second, and perhaps more important, there is a question of whether all spikes or just bursts should be considered when analyzing spike data. It has been argued that hippocampal synapses filter out single spikes as if they were noise, but transmit bursts reliably
(Lisman 1996). This raises the possibility that the important signals are carried by bursts. If this is true, plots of burst phase versus position will reveal more precise timing and hence less variability in the phase data.

PHASE, NOT FIRING RATE, CODES FOR ACTUAL LOCATION

Our model implies that in recall mode, place cell firing early in the theta cycle codes for actual location, whereas cells firing with late phase are predictors of future position. Because prediction is necessarily risky, cells firing early in the theta cycle will be better indicators of actual position than cells firing late in the theta cycle. This has been confirmed experimentally by Skaggs et al. (1996). It has also been observed that cells firing early or late in the theta cycle have a lower firing rate than cells firing on the peak of the theta cycle (where there can be multiple spikes per gamma cycle). This implies that the place cells with the highest firing rate are not the cells coding for the actual location. Given the simplified way we have approximated spike firing, our model is not able to account for data in which multiple spikes (bursts) occur in gamma cycles. An extension of our model with more realistic bursting neurons is being developed for this and other phenomena: the observation that the phase advance approaches $360^{\circ}$ for strong place fields (in our model, only $200^{\circ}$, Fig. 3) and data regarding two-dimensional place fields.

\section{TIMING OF INPUTS TO THE CA3 REGION}

We have assumed that during recall, the CA3 system is cued by direct sensory inputs early in the theta cycle. Some support for this comes from the work of Skaggs et al. (1996), showing that firing in the dentate preceeds firing in $\mathrm{CA} 3$ by $90^{\circ}$. One difficulty with reconciling the model with the data, however, is that the phase precession observed in CA3 and CA1, which we have attributed to process in CA3, can also be observed in the dentate. For this to occur there would have to be firing during multiple gamma cycles in the dentate. We do not yet have a clear explanation for this. One possibility suggested by Buzsáki (pers. comm.) is that part of the activity in dentate reflects synaptic feedback from CA3. As suggested by the results in Figure 2 , it is desirable to

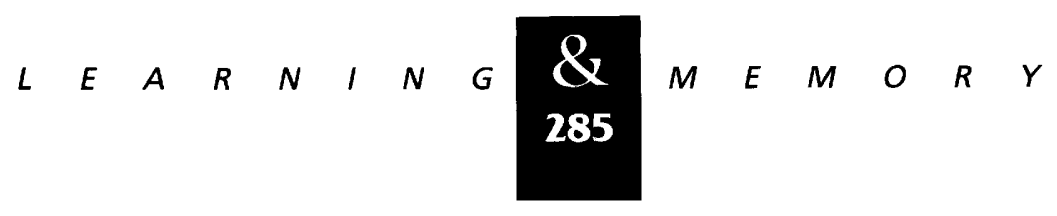


cue the sequence during recall with the first two items in the sequence, not just with one. The reason has to do with the cumulative nature of NMDA-mediated readout described in Jensen and Lisman (1996b, this issue). Briefly, multiple cuing is desirable because it eliminates ambiguity when two paths cross. The idea that multiple cues are used to stimulate the recall of the full sequence can be rephrased to say that a partial sequence is used to recall the full sequence. Because the slow NMDA channels necessary to store sequence information are present not only in hippocampus, but also in cortex, it is possible that the cortex itself stores partial sequences, that current input stimulates the cortex to produce a partial sequence, and that the input of the partial sequence into the CA3 region of hippocampus then produces the full sequence.

\section{COMPARISON WITH OTHER MODELS}

With regard to specifically predicting the phase advance of O'Keefe and Reece (1993) (Fig. 2), our work has important similarities with the model of Tsodyks et al. (1996). As in our model, hippocampal cells that fire early in the theta cycle are driven by current sensory input, and these cells, in turn, stimulate other CA3 cells to fire late in the theta cycle. What is novel in our model are the strong constraints imposed by network oscillations, and the use of known conductances [NMDA, afterhyperpolarization (AHP)] in accounting for network function. It is some of these further assumptions that allow quantitative prediction of place-cell data and the value of the phase advance. In the model of Tsodyks et al. (1996) the speed of sequence readout is determined by adjustable parameters of the model. In our model the magnitude of phase advance is accounted for with no free parameters.

\section{THE HIPPOCAMPUS SITS AT THE APEX OF THE INFORMATION FLOW IN THE BRAIN}

Anatomical work shows that the hippocampus sits at the apex of communication flow in the brain. Sensory data from specialized cortical areas converges onto polymodal association areas that then provide input to the hippocampus. Interestingly, information is also sent from the hippocampus back to the polymodal areas, which in turn sends information back to the specialized sensory areas. The fact that the hippocampus is able to use current information and stored sequences to predict what is expected in the future raises the question of what will be done with these predictions. The existence of pathways for flow of information back to cortical areas suggests that these predictions are sent to the cortex, where expectations are set. We refer the reader to various theoretical papers on how these expectancies might be used by the cortex (Mumford 1994; W. Softky, pers. comm.).

\section{Acknowledgments}

We thank Ilan Golani and Robert Muller for helpful discussions and Larry Abbott and Jean-Marc Fellous for reading the manuscript. This work was supported by the W.M. Keck Foundation, a National Institutes of Health grant (NS 27337), and the Alfred P. Sloan Foundation (94-10-1).

The publication costs of this article were defrayed in part by payment of page charges. This article must therefore be hereby marked "advertisement" in accordance with 18 USC section 1734 solely to indicate this fact.

\section{References}

Blum, K.I. and L.F. Abbott. 1996. A model for spatial map formation in the hippocampus of the rat. Neur. Computat. 8: 85-93.

Buzaśki, G. 1989. Two-stage model of memory trace formation: A role for "noise" brain states. Neuroscience 31: 551-570.

Debanne, D., N.C. Guerineau, B.H. Gahwiler, and S.M. Thompson. 1995. Physiology and pharmachology of unitary synaptic connections between pairs of cells in areas CA3 and CA1 of rat hippocampal slice cultures. I. Neurophysiol. 73: 1281-1294.

Eilam, D. and I. Golani. 1989. Home base behavior of rats (Rattus norvegicus) exploring a novel environment. Behav. Brain Res. 34: 199-211.

Golani, I., Y. Benjamini, and D. Eilam. 1993. Stopping behavior: Constraints on exploration in rats (Rattus norvegicus). Behav. Brain Res. 53: 21-33.

Granger, R., J. Whitson, J. Larson, and G. Lynch. 1994. Non-Hebbian properties of long-term potentiation enable high-capacity encoding of temporal sequences. Proc. Natl. Acad. Sci. 91: 10104-10108.

Hasselmo, M.E. and J.M. Bower. 1993. Acetylcholine and memory. Trends Neurosci. 16: 218-222.

Jensen, O. and J.E. Lisman. 1996a. Novel lists of $7 \pm 2$ known items can be reliably stored in an oscillatory 
short-term memory network: Interaction with long-term memory. Learn. \& Mem. (this issue).

1996b. Theta/gamma networks with slow NMDA channels learn sequences and encode episodic memory: Role of NMDA channels in recall. Learn. \& Mem. (this issue).

Jensen, O., M.A.P. Idiart, and J.E. Lisman. 1996.

Physiologically realistic formation of autoassociative memory in networks with theta/gamma oscillations: Role of fast NMDA channels. Learn. \& Mem. (this issue).

Lisman, J.E. 1996. Bursts as a unit of neural information. Trends. Neurosci. (in press).

Lisman, J.E. and M.A.P. Idiart. 1995. Storage of $7 \pm 2$ short-term memories in oscillatory subcycles. Science 267: 1512-1515.

McNaughton, B.L., C.A. Barnes, and J. O'Keefe. 1983. The contributions of position and velocity to single unit activity in the hippocampus of freely-moving rats. Exp. Brain Res.

52: $41-49$.

Manai, A.A. and W.B. Levy. 1993. The dynamics of sparse random network. Biol. Cybernet. 70: 177-187.

Muller, R.U. and J.L. Kubie. 1989. The firing of hippocampal place cells predicts the future position of freely moving rats. I. Neurosci. 9: 4101-4110.

Muller, R.U., J.L. Kubie, and J.B. Ranck. 1987. Spatial firing patterns of hippocampal complex-spike cells in a fixed environment. J. Neurosci. 7: 1935-1950.

Mumford, D. 1994. Neuronal architectures for pattern-theoretic problems. In Large scale neuronal theories of the brain (ed. C. Koch and J.L. Davies), pp. 125-152. MIT Press, Cambridge, MA.

O'Keefe, J. and J. Dostrovsky. 1971. The hippocampus as a spatial map: Preliminary evidence from unit activity in freely moving rats. Brain Res. 34: 171-175.

O'Keefe, J. and M.L. Recce. 1993. Phase relationship between hippocampal place units and the EEG theta rhythm. Hippocampus 3: 317-330.

Olton, D.S., M. Branch, and P.J. Best. 1978. Spatial correlates of hippocampal unit activity. Exp. Neurol. 58: $387-409$.

Prepscius, C. and W.B. Levy. 1994. Sequence prediction and cognitive mapping by a biological plausible neural network. Proc. INNS World Congress on Neural Networks IV: $164-169$.

Skaggs, W.E. and B.L. McNaughton. 1996. Replay of neuronal firing sequences in rat hippocampus during sleep following spatial experience. Science 271: 1870-1873.

Skaggs, W.E., B.L. McNaughton, M.A. Wilson, and C.A. Barnes. 1996. Theta phase precession in hippocampal neuronal populations and the compression of temporal sequences. Hippocampus 6: 149-172.

Tchernichovski, O. and I. Golani. 1995. A phase plane representation of rat exploratory behavior. J. Neurosci. Methods 62: 21-27.

Tsodyks, M.V., W.E. Skaggs, T.J. Sejnowski, and B.L. McNaughton. 1996. Population dynamics and theta rhythm phase precession of hippocampal place cell firing: A spiking neuron model. Hippocampus (in press).

Received June 26, 1996; accepted in revised form September 25,1996 


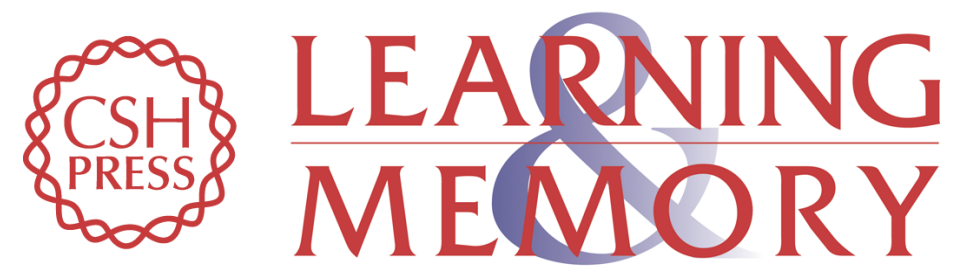

\section{Hippocampal CA3 region predicts memory sequences: accounting for the phase precession of place cells.}

O Jensen and J E Lisman

Learn. Mem. 1996, 3:

References This article cites 18 articles, 5 of which can be accessed free at: http://learnmem.cshlp.org/content/3/2-3/279.full.html\#ref-list-1

License

Email Alerting

Service

Receive free email alerts when new articles cite this article - sign up in the box at the top right corner of the article or click here. 